\section{$\underset{\substack{\text { hommes } \\ \text { \& migrations }}}{ }$}

\section{Hommes \& migrations}

Revue française de référence sur les dynamiques

migratoires

\section{$1291 \mid 2011$}

Diasporas sri lankaises

\title{
L'immigration sri lankaise
}

\section{L'immigration sri lankaise}

\author{
Regards croisés
}

\section{Anthony Goreau-Ponceaud}

\section{(2) OpenEdition}

\section{Journals}

\section{Édition électronique}

URL : http://journals.openedition.org/hommesmigrations/666

DOI : 10.4000/hommesmigrations.666

ISSN : 2262-3353

\section{Éditeur}

Musée national de l'histoire de l'immigration

Édition imprimée

Date de publication : 1 mai 2011

Pagination : 6-10

ISSN : 1142-852X

Référence électronique

Anthony Goreau-Ponceaud, «L'immigration sri lankaise », Hommes \& migrations [En ligne], 1291 | 2011, mis en ligne le 31 décembre 2013, consulté le 30 avril 2019. URL : http://journals.openedition.org/ hommesmigrations/666 ; DOI : 10.4000/hommesmigrations.666 


\section{L'immigration sri lankaise Regards croisés}

Par Anthony Goreau-Ponceaud, géographe, maître de conférences à l'université de Bordeaux-IV, UMR ADES CNRS

Sri Lanka, île de l'océan Indien de plus de 20 millions d'habitants, dont les trois quarts ont pour langue maternelle le cinghalais (une langue issue du fonds indo-aryen) et le dernier quart le tamoul (langue dravidienne d'Inde du Sud), a été marqué pendant près de trois décennies par un conflit séparatiste sanglant, déclenché par des groupes militants appartenant à la minorité tamoule autochtone. Ce conflit a eu de larges répercussions dans les logiques migratoires des Sri Lankais : terre d'immigration depuis les débuts de son histoire, Sri Lanka est devenu progressivement au cours des années soixante-dix une terre d'émigration massive. Si des phénomènes limités d'émigration s'étaient déjà produits dans le cadre du système colonial, ils étaient sans aucune mesure avec ceux qui se sont mis en place après 1948, date à laquelle Sri Lanka a accédé à son indépendance. À partir de ce moment charnière de l'histoire de l'île, la minorité tamoule (13\%, dont $8 \%$ de Tamouls sri lankais), choyée par les colonisateurs, se retrouve soumise à la loi de la majorité cinghalaise (74\%), qui impose sa langue - le cinghalais - et donne la prééminence à sa religion, le bouddhisme. La mise en place de lois discriminantes a lancé sur les routes de l'exil, de manière sporadique dans un premier temps, des élites anglophones vers les pays du Commonwealth. Après les pogroms anti-tamouls de juillet 1983, correspondant également à la montée en puissance du mouvement des Tigres de libération de l'Eelam tamoul (Liberation Tigers of Tamil Eelam - LTTE), les flux de réfugiés et de demandeurs d'asile en direction des pays du Nord, d'Asie(Malaisie et Inde en particulier) et du Moyen-Orient deviennent significatifs.

Si cette émigration peut être considérée comme une conséquence de la décolonisation britannique, elle n'est pas classique pour autant: c'est sous l'effet des lois britanniques (1962, 1968 et particulièrement 1971) restreignant l'immigration des citoyens du Nouveau Commonwealth, que les comportements migratoires des populations originaires de Sri Lanka se sont quelque peu modifiés. C'est ainsi que les Sri Lankais, majoritairement tamouls (en cela cette immigration ne reflète pas la diversité ethnolinguistique de la population de l'île composée de Tamouls sri lankais et indiens, de Cinghalais, de Burghers, de Malais et de Veddas ${ }^{(1)}$, se sont progressivement tournés 
vers la France, l'Allemagne, la Suisse, l'Italie, les Pays-Bas et la Norvège pour l'Europe, mais également vers les États-Unis et surtout vers le Canada (Toronto en particulier) pour le Nouveau Monde ${ }^{(2)}$. Cette immigration n'est pas classique, également, parce qu'elle s'inscrit dans une histoire tourmentée marquée par une guerre civile cruelle et meurtrière, à l'origine d'une importante diaspora politiquement active. Les organisations politiques tamoules évaluent d'ailleurs à plus d'un million le nombre de Tamouls sri lankais vivant hors de Sri Lanka. Comme le précise Éric Meyer dans ce dossier, "Sri Lanka est probablement, dans la première décennie du XXI" siècle, le pays d'Asie dont la plus forte proportion de la population vit de façon temporaire ou durable en dehors de ses frontières".

En mobilisant les contributions de chercheurs, enseignants chercheurs, doctorants et postdoctorants français et étrangers (norvégien, suisse, canadien et britannique), il s'agit dans ce dossier de renouveler la production de connaissances concernant cette immigration. Les premières analyses de l'immigration sri lankaise en France remontent à la décennie quatre-vingt-dix (songeons en particulier aux travaux pionniers de Gérard Robuchon, Marie Percot et Andrea Tribess). Elles ont été partiellement complétées par celles d'Éric Meyer, Angelina Étiemble et Jean-Michel Delage.

Ce renouvellement apparait comme une nécessité au regard de l'évolution de nos sociétés de plus en plus confrontées au multiculturalisme et au pluralisme religieux. Ces tensions donnent à penser que nos sociétés, aujourd'hui, peinent à assurer leur intégration, idée qui peut se prolonger par celle d'une

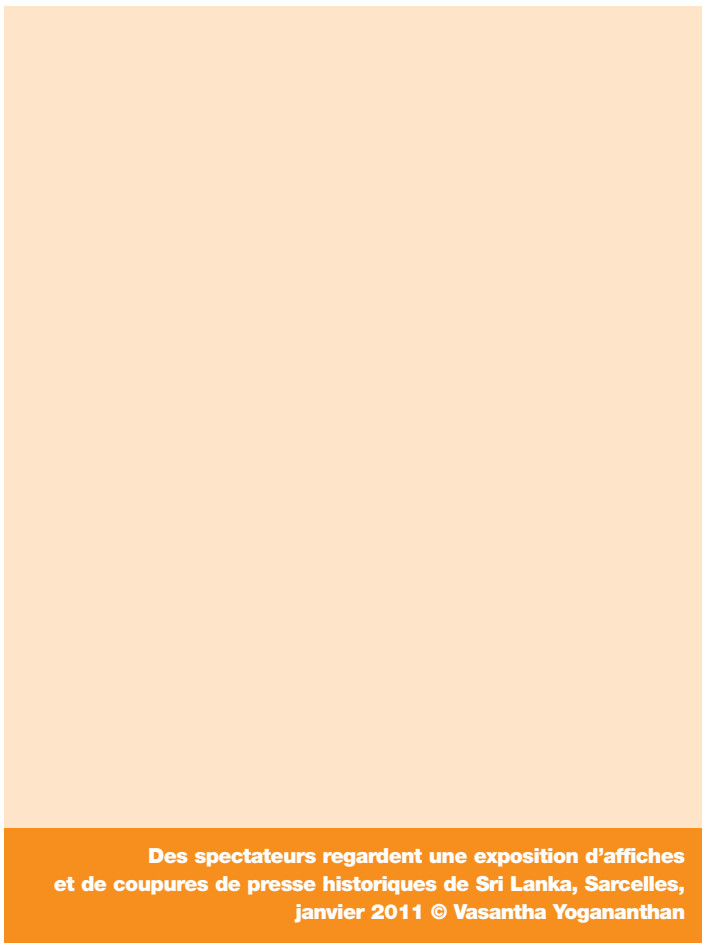
crise du concept même d'intégration - un concept des sciences sociales qui a longtemps été associé à l'image d'une forte correspondance entre la société, l'État et la nation, et auquel est fréquemment lié un autre concept : la socialisation. 
En multipliant les angles d'analyse, les approches disciplinaires (géographie, anthropologie, sciences politiques, histoire, ethnologie et science des religions) et les terrains d'étude, entre la France, la Suisse, la Norvège, le Canada et la Malaisie, l'objet de ce numéro est de montrer qu'il existe, comme le suggère Arjun Appadurai, de nouvelles “communautés imaginées", dont les contours sont problématiques et l'espace planétaire, et non plus national.

C'est d'autant plus vrai qu'une grande part de cette immigration se revendique ellemême comme formant une diaspora, dont les contours sont certes discutables, mais au sein de laquelle la question du chez-soi se pose avec acuité. À partir des analyses de Philippe Gazagne, Christopher McDowell, Stine Bruland Sørensen, Pierre-Yves Trouillet et Mark Bradley, on se rend rapidement compte que le migrant, ou le diasporé, n'est pas doublement absent au lieu d'origine et d'arrivée (comme le suggère Abdelmalek Sayad $\left.{ }^{(3)}\right)$, mais doublement présent ${ }^{(4)}$. Ce qui assure cette continuité dans le cadre de la diaspora tamoule de Sri Lanka, c'est tout à la fois un projet politique, l'Eelam tamoul, des instances politiques transnationales, la religion, et un passé idéalisé voire essentialisé, comme le précise Gaëlle Dequirez dans ce dossier. Tous ces éléments participent à la reconstruction d'une identité "déracinée" et génèrent une image, parfois trompeuse, d'une vie privée qui demeure dans une large mesure confinée à la communauté. D'autant plus qu'un ensemble d'associations et d'activités plus ou mois liées à la lutte pour l'Eelam tamoul dominent la vie sociale. Ce qui semble pour les différents auteurs un trait caractéristique de cette immigration aussi bien en France que dans l'ensemble de l'espace diasporique.

En interrogeant, en toile de fond, les "modèles d'intégration" des différents pays d'accueil, ces regards croisés permettent d'envisager les interrogations et les crispations sur l'identité nationale que suscite ce segment migratoire dans les différents pays d'installation. D'autant que le simple fait d'appartenir à une diaspora inscrit des demandes de reconnaissance dans un espace à la fois politique et géopolitique qui ne correspond qu'en partie à l'espace national du pays considéré. Dès lors, comment tenir compte, dans des politiques qui se veulent d'intégration et de socialisation, de ces phénomènes diasporiques, qui peuvent véhiculer des demandes culturelles ou mémorielles particulières?

Les Tamouls sri lankais réclament le droit d'apparaître en pleine lumière, à travers des manifestations religieuses ou politiques. Les contributions de Philippe Gazagne, de Giacomo Mantovan, de Brigitte Tison et de Véronique Bouillier nous montrent dans une certaine mesure que le défi reste, pour la société française, de reconstruire une théorie de l'universalisme démocratique fondé sur le besoin de reconnaissance des identités individuelles et collectives. Ces questionnements, en suivant l'analyse de Delon Madavan, trouvent un écho similaire dans d'autres pays comme la Malaisie. 
En interrogeant également le couple patrimoine-migration, les auteurs de ce numéro questionnent les pratiques des migrants sri lankais et leurs interactions avec les politiques migratoires permettant d'aborder la question du pouvoir : différenciation (entre les groupes sri lankais eux-mêmes et avec le groupe majoritaire) et hiérarchisation sociale (autour des processus de catégorisation ethnique, de visibilité et de mobilisation politique). Il est vrai que ce qui se joue en la matière pour les migrants varie considérablement d'un pays à un autre. Dans certains pays, leur intégration sociale est encouragée, alors que toute reconnaissance culturelle est exclue et que leur participation à la vie civique et civile est réduite à la portion congrue. En conjuguant ces deux thèmes, les auteurs contribuent à mettre au jour l'aura d'essentialisme qui entoure trop souvent la notion de patrimoine, pour explorer cette dernière plutôt sous l'angle de la contemporanéité diverse et mobile. Mémoire et oubli s'expriment non seulement à travers les récits de demande d'asile, les souffrances de l'exil, mais aussi dans la sélection d'événements qui deviennent "patrimoine" et participent à la fabrication d'une historiographie du côté de la diaspora (songeons par exemple au festival de Ganesh en France). On pourrait d'ailleurs s'interroger sur la façon dont ce patrimoine concerne non seulement un groupe particulier mais aussi sa place dans une société ethniquement diverse. En cela, Vasantha Yogananthan, en photographiant des mises en scène de la communauté tamoule sri lankaise en France, essaie de montrer comment la diaspora tamoule, bercée par une iconographie du conflit, reproduit et transforme les mythes et images de sa propre histoire ${ }^{(5)}$.

Notons encore que le patrimoine concerne la dynamique temporelle évoquée par des termes tels que "mémoire" ou "souvenir", et aussi la dimension spatiale, puisqu'il crée de nouveaux territoires. En cela, Philippe Gazagne nous offre une analyse originale du quartier de La Chapelle en tant que portion d'un vaste espace diasporique et nous montre que les réseaux de solidarité qui s'y déploient, de façon transnationale, peuvent finalement être contraignants, ce qui complexifie ainsi les discours sur les solidarités. Ces contributions montrent que la multiplication contemporaine des référentiels identitaires, loin de "déraciner" l'individu ou le groupe en quête de sens, le contraignent plutôt à rechercher une cohérence sociale et spatiale autour de son histoire et de la construction de sa propre territorialité (de plus en plus diversifiée, sinon mobile, il est vrai), et pose avec acuité la question de l'habiter, du home, pour des populations souvent à la fois ici, là-bas et ailleurs, prises en étau par une double ambivalence entre ancrage et mobilité, ouverture et fermeture ${ }^{(6)}$. Enfin, les auteurs de ce numéro nous rappellent que les identités individuelles et collectives, résultats d'élaborations sociales et culturelles, s'avèrent d'autant plus solides qu'elles transitent malgré tout par le langage matériel de l'espace, de ses lieux et de ses territoires. 


\section{Bibliographie}

- Appadurai Arjun. Après le colonialisme. Les conséquences culturelles de la globalisation, Paris, Payot-Rivages, 2001, 322 p.

- Dequirez Gaëlle, "Tamouls sri lankais: le Little Jaffna de La Chapelle”, in Hommes \& Migrations, n 1268-1269, 2007, pp. 82-91.

- Delage Jean-Michel, Vanakam. Les Tamouls Sri Lankais en France, Livry-Gargan, Castor \& Pollux, 2004, 128 p.

- Étiemble Angélina, Les Ressorts de la diaspora tamoule en France : associations, médias, politique, Rennes, Aderiem/Mire, 2001, 169 p.

- Étiemble Angélina, "Les Tamouls sri lankais dans la région parisienne : la politisation du communautaire", in Les Cahiers du CERIEM, 2001, n 7, pp. 19-32.

- Meyer Éric, Sri Lanka, entre particularismes et mondialisation. Paris, La Documentation française, 2001, 183 p.

- Moudiappanadin Joseph, "Tamouls, une communauté plurielle", in Accueillir, 1993, n 191, pp. 26-27.

- Moudiappanadin Joseph, L'Immigration tamoule en France, Paris, CHEAM, 1997, 45 p.

- Robuchon Gérard, Percot Marie, Tribess Andrea, "Tamouls sri lankais en France", Rapport pour le ministère de la Ville et des Affaires sociales (direction de la population et des migrations), Paris, 1995, 225 p.

- Robuchon Gérard, "Étagères à bons dieux. Autels domestiques tamouls en immigration", in Ferveurs contemoraines Textes d'anthropologie urbaine offerts à Jacques Gutwirth, réunis par Colette Pétonnet et Yves Delaporte, Paris, L'Harmattan, collection "Connaissance des hommes", 1993, pp. 127-138. En ligne : http://halshs.ccsd.cnrs.fr/halshs-00003996

- Robuchon Gérard, "Pratiques sociales et pratiques religieuses des Tamouls au Sacré-Cceur de Paris", in Piault MarcHenri (dir.), Vers des sociétés pluriculturelles : études comparatives et situation en France, Paris, ORSTOM, 1987, pp. 332-336.

- Vuddamalay Vasoodeven, "Présence indienne en France. Les facettes multiformes d'une immigration invisible", in Revue européenne des migrations internationales, vol. 5, n³ 3, 1989, pp. 65-77.

\section{Notes}

1. Dans la société sri lankaise se juxtaposent des communautés qui diffèrent par la langue, la religion et les structures sociales. D'origine indo-aryenne (du nord de l'Inde) et à grande majorité bouddhiste, la communauté cinghalaise est la population majoritaire dans le pays. La population tamoule, d'origine dravidienne (sud de l'Inde) est scindée en deux ensembles : les Tamouls "autochtones" et les Tamouls des plantations, qui ont un statut et des intérêts très différents. Les Tamouls "autochtones", présents bien avant la colonisation aux côtés des Cinghalais, ont le sentiment d'être, tout comme ces derniers, des "enfants du sol" et non des immigrés. Les Tamouls des plantations, arrivés au XIX siècle, ont été utilisés, sous la domination britannique, comme main-d'ceuvre dans les plantations du centre du pays, enclavées dans des régions à forte majorité cinghalaise. Les Malais quant à eux forment un groupe important évalué à 50000 membres. Ce sont des descendants de Javanais exilés par les Hollandais (de 1658 à 1796) pour des raisons politiques. Deux autres groupes tiennent un rôle de plus en plus marginal dans le pays en raison de leur faiblesse numérique. D’une part les Burghers, qui sont les descendants de colons européens (principalement portugais, néerlandais, allemands et britanniques arrivés suivant les différentes phases migratoires entre les $\mathrm{XVI}^{\mathrm{c}}$ et $\mathrm{XX}^{\mathrm{e}}$ siècles) ou d'unions mixtes entre colons et femmes indigènes, avec des minorités de Suédois, Norvégiens, Français et Irlandais. Dans leur majorité, ils ont préféré quitter le pays pour les grands États membres du Commonwealth à la suite de la politique de cinghalisation menée par le gouvernement de Colombo à partir de 1956. Les quelques Burghers qui restent dans l'île ne gardent qu'un rôle économique et se concentrent surtout dans la capitale. D'autre part, les Veddas (ou Wanniyala-Aetto), qui étaient les premiers habitants de l'île et qui vivent dans les forêts, ne sont plus que quelques centaines d'individus (rassemblés dans le parc national de Maduru Oya) et tendent à perdre leur identité.

2. Il sera d'ailleurs exclusivement question dans ce numéro de l'immigration tamoule sri lankaise. Cela peut apparaitre comme une limite, mais c'est surtout révélateur de la visibilité de ce segment migratoire, au détriment de l'immigration cinghalaise, peu étudiée car peu visible.

3. Abdelmalek Sayad, La Double Absence. Des illusions de l'émigré aux souffrances de l'immigré, Paris, Seuil, coll. "Liber", 1999,437 p.

4. Comme l'a montré notamment Dana Diminescu, à travers les NTIC, les migrants d'aujourd'hui sont les acteurs d'une culture de/du lien, qu'ils ont eux-mêmes fondée et qu'ils entretiennent dans la mobilité.

5. Le présent dossier est illustré par les clichés de Vasantha Yogananthan.

6. Si Bourdieu précise dans la préface de La Double Absence d'Abdelmalek Sayad, que "limmigré est atopos, sans lieu, déplacé, inclassable l... ni vraiment du côté du Même, ni totalement du côté de l'Autre, il se situe en ce lieu 'bâtard' dont parle aussi Platon, la frontière de l'être et du non être social', on pourrait ajouter que le diasporé, lui, habite la mobilité, une sorte d'éther diasporique. 